\title{
Amplitude of low-frequency fluctuation may be an early predictor of delayed motor development due to neonatal hyperbilirubinemia: a fMRI study
}

\author{
Kai Yan ${ }^{1 \#}$, Feifan Xiao" ${ }^{1 \#, ~ Y u w e i ~ J i a n g ~}{ }^{2 \#}$, Chunmei Lu ${ }^{1}$, Yong Zhang ${ }^{3}$, Yanting Kong ${ }^{1}$, Jian Zhou ${ }^{4}$, \\ Junbo Wang ${ }^{4}$, Chengxiang Lin ${ }^{4}$, Haowei Yang ${ }^{4}$, Dajiang Zhang ${ }^{4}$, Guoqiang Cheng ${ }^{1}$, Zhongwei Qiao ${ }^{4}$, \\ Liping Wang', Qian Qin ${ }^{5}$, Wenhao Zhou'
}

${ }^{1}$ Department of Neonatology, Children' Hospital of Fudan University, Shanghai, China; ${ }^{2}$ Institute of Neuroscience, Key Laboratory of Primate Neurobiology, CAS Center for Excellence in Brain Science and Intelligence Technology, Chinese Academy of Sciences, Shanghai, China; ${ }^{3}$ MR Research, GE Healthcare, Shanghai, China; ${ }^{4}$ Department of Radiology, Children' Hospital of Fudan University, Shanghai, China; ${ }^{5}$ Children's Hospital of Fudan University, Shanghai Key Laboratory of Birth Defects, The Translational Medicine Center of Children Development and Disease of Fudan University, Shanghai, China

Contributions: (I) Conception and design: K Yan, Z Qiao, W Wang, Q Qin, W Zhou; (II) Administrative support: K Yan, W Zhou; (III) Provision of study materials or patients: None; (IV) Collection and assembly of data: F Xiao, Y Jiang, C Lu, Y Zhang, Y Kong, J Zhou, J Wang, C Lin, H Yang, D Zhang, G Cheng; (V) Data analysis and interpretation: K Yan, Y Jiang, Q Qin; (VI) Manuscript writing: All authors; (VII) Final approval of manuscript: All authors.

"These authors contributed equally to this work and are co-first authors.

Correspondence to: Wenhao Zhou. Children's Hospital of Fudan University, 399 Wanyuan Road, Shanghai 201102, China.

Email: zhouwenhao@fudan.edu.cn; Zhongwei Qiao. Children’s Hospital of Fudan University, 399 Wanyuan Road, Shanghai 201102, China.

Email: qiaozhwei@163.com; Liping Wang. CAS Center for Excellence in Brain Science and Intelligence Technology, Chinese Academy of Sciences, Shanghai, China. Email: liping.wang@ion.ac.cn; Qian Qin. Children’s Hospital of Fudan University, 399 Wanyuan Road, Shanghai 201102, China.

Email: qinqian89@outlook.com.

Background: Acute bilirubin encephalopathy or kernicterus is the worst consequence of brain damage caused by the elevation of total unbound serum bilirubin (TSB) in neonates. The present study aimed to visualize the characteristic brain regions of neonates with hyperbilirubinemia $(\mathrm{HB})$ using functional magnetic resonance imaging (fMRI) and to measure the amplitude of low-frequency fluctuation (ALFF) values.

Methods: This was a prospective cohort study, which included newborns with HB who were hospitalized at the Children's Hospital of Fudan University. The control group included neonates admitted with neonatal simple wet lung or pneumonia without neurological disease or brain injury. Newborns were divided into a severe hyperbilirubinemia group (SHB), moderate HB group, and control group based on TSB levels. The newborns completed routine MRI combined with fMRI scans and brainstem auditory evoked potentials (BAEPs) during their hospitalization.

Results: A total of 251 newborns were included in this study. There were 45 patients in the SHB group, 65 in the HB group, and 141 in the control group. The average ALFF value in the basal ganglia region in the SHB group was the highest, which was greater than that in the $\mathrm{HB}$ and control groups $(\mathrm{P}<0.001)$. The ALFF increased with an increase in TSB concentration. Based on the results of the Bayley Scales of infant development assessment, we further found that the most significant difference in ALFF remained in the basal ganglia region between newborns with motor development scores above 70 (including 70) and below 70. Correlation analysis revealed a strong negative correlation between motor development scores and ALFF $(\mathrm{r}=-0.691, \mathrm{P}<0.001)$. When ALFF alone was used to predict motor development, the sensitivity was $89 \%$. When ALFF was combined with TSB and BEAP results, the area under the ROC curve was the largest (AUC $=0.85$ ). The sensitivity and specificity of the model were $67.86 \%$ and $90.77 \%$, respectively.

Conclusions: The ALFF value may be able to serve as an early imaging biomarker and has a greater sensitivity than TSB or BAEP results in predicting long-term motor development $(18 \mathrm{~m})$ in HB. 


\begin{abstract}
Keywords: Hyperbilirubinemia (HB); neonate; amplitude of low frequency fluctuation; functional magnetic
\end{abstract} resonance imaging (fMRI)

Submitted Dec 15, 2020. Accepted for publication Mar 15, 2021.

doi: $10.21037 / \mathrm{tp}-20-447$

View this article at: http://dx.doi.org/10.21037/tp-20-447

\section{Introduction}

Acute bilirubin encephalopathy or kernicterus is the worst consequence of brain damage caused by the elevation of total serum bilirubin (TSB) in neonates (1), which may lead to severe neurological sequelae in the later stage (2-4). The TSB concentration is markedly elevated (over $20 \mathrm{mg} / \mathrm{dL}$ ) over a short period of time in newborns, which may result in clinical evidence of brain damage, ranging from subtle neurologic abnormalities to severe encephalopathy or permanent neurologic damage to death (5). Therefore, in neonates with hyperbilirubinemia (HB), the TSB concentration is an important routine indicator to determine the severity of the disease and requires regular monitoring of the concentration.

At present, brain injury caused by a high concentration of TSB usually needs to be confirmed by routine magnetic resonance imaging (MRI). Studies have reported that T1 signal increases appeared in the bilateral regions of the pallidum and sub-thalamic nucleus on routine MRI within the first seven days after birth in neonates with acute bilirubin encephalopathy (6), and T2 hyperintensity was found in kernicterus or chronic bilirubin encephalopathy (7). However, these manifestations are still not considered the gold standard for the diagnosis of bilirubin encephalopathy. Neuropathological studies have shown that large amounts of microglia can be found in basal ganglia areas in vitro and in animal models of kernicterus $(8,9)$. However, the significance of these neuronal proliferations is unclear. Brainstem auditory evoked potentials (BAEPs) $(3,10-14)$ and routine MRI provide only qualitative findings and do not have a high specificity in the development of long-term bilirubin encephalopathy or poor neurological prognosis. In the diagnosis of bilirubin encephalopathy, there is an urgent need for quantitative biological parameters that allow rapid assessment of neuroimaging changes at an early stage.

Compared to routine MRI, functional magnetic resonance imaging (fMRI) is also a non-invasive, nonradioactive technique for observing brain activity and has been widely used in neuroscience and cognitive science $(15,16)$. Amplitude of low-frequency fluctuation (ALFF) is a relatively stable indicator in $\mathrm{fMRI}$ sequences that can respond to the low-frequency electrical activity of local neurons in the resting state. Most studies using ALFF as a biomarker have focused on specific brain region findings and functional connectivity in patients with psychiatric and neurodegenerative disorders (17-23). ALFF has the potential to be a special tool in the study of psychiatric or neurological disorders to assess the electrical activity of neurons in the resting state early in the course of the disease $(24,25)$. The present study was designed to visualize the characteristic brain regions of hyperbilirubinemic neonates using $\mathrm{fMRI}$ and to measure the ALFF values in specific brain regions in the resting state. We will use ALFF values in combination with serum total bilirubin values and BAEP results to predict neurobehavioral developmental outcomes in neonates at 18 months of age. We present the following article in accordance with the STROBE reporting checklist (available at http://dx.doi.org/10.21037/tp-20-447).

\section{Methods}

\section{Study design}

This was a prospective cohort study. The study selected ALFF as a neonatal exposure factor with the purpose of observing the long-term neurodevelopmental status (18 months old) of neonates with HB at different postmenstrual ages (PMA). The study included newborns with HB who were hospitalized in the Department of Neonates, Children's Hospital of Fudan University between November 2016 and December 2017. These neonates were hospitalized for HB. The control group included neonates admitted with neonatal simple wet lung or pneumonia without HB during hospitalization, neurological disease, or brain injury. The newborns underwent routine MRI and fMRI scans during hospitalization. Newborns with serum total bilirubin above the exchange line were included in the severe hyperbilirubinemia group (SHB); those with serum total bilirubin values above the phototherapy line but below the exchange line were included in the moderate HB group, and the control group consisted of newborns with simple 
wet lung or pneumonia (control).

The inclusion criteria were as follows: (I) gestational age of 37 weeks or more for full-term babies; (II) diagnosis of neonatal HB based on postnatal age with reference to the bilirubin reference curve of Bhutani et al. (1,26-29); (III) the guardian of the newborn signed the informed consent for examination and clinical treatment.

The exclusion criteria were as follows: (I) newborns who were more than 28 days old or who had already been treated in another hospital. (II) Newborns suffering from other neurological diseases: (i) suspected or diagnosed congenital structural malformation of the central nervous system; (ii) the diseases that cause acute significant brain injury, including brain tumors, cerebral hemorrhage, hydrocephalus, hypoxic-ischemic encephalopathy, meningitis, and other central nervous system infections; $d$. traumatic brain injury. (III) The time from the discovery of $\mathrm{HB}$ to completion of the MR scan was longer than seven days. (IV) The data quality does not meet the requirements of research and analysis: (i) sudden change in the condition; (ii) during the scan, the newborn woke up and produced a large number of motor artifacts, which could not be used for reporting and analysis.

The study protocols were registered at www.clinicaltrial. gov (NCT02544100) and were approved by the Medical Ethics Committee of Children's Hospital of Fudan University (2020-247). Written informed consent was obtained from the parents of the infant participants involved in our study. The study was conducted in accordance with the Declaration of Helsinki (as revised in 2013).

\section{Clinical baseline and laboratory data}

Baseline data included sex, PMA, birth weight, hypertension during pregnancy, hyperglycemia during pregnancy, thyroid dysfunction during pregnancy, parental hepatitis, 5 min Apgar scores, 10 min Apgar scores, routine MR test results (abnormal signals in the basal ganglia or subthalamic region), neonatal hemolytic disease, TSB, and BAEP results. All demographic and clinical information were obtained from the HIS clinical information system.

Neonates with HB were admitted to the hospital for phototherapy, and serum bilirubin levels were monitored. Transcutaneous bilirubin was monitored every $12 \mathrm{~h}$ for the first $96 \mathrm{~h}$ after phototherapy. If transcutaneous bilirubin exceeded the phototherapy line, we continued phototherapy and monitored the TSB every 24 hours until the TSB dropped below the phototherapy line and did not fluctuate.

\section{Phototherapy treatment}

According to the phototherapy reference curve $(27,30)$ and phototherapy method (31), all neonates with HB received standard intensity phototherapy $\left[8-10 \mu \mathrm{W} /\left(\mathrm{cm}^{2} \cdot \mathrm{nm}\right)\right]$ when their serum total bilirubin values exceeded the phototherapy threshold. When the serum total bilirubin value reached or exceeded the threshold of blood exchange, they received continuous strong phototherapy for 4-6 h $\left[30 \mu \mathrm{W} /\left(\mathrm{cm}^{2} \cdot \mathrm{nm}\right)\right]$, and when the serum total bilirubin decreased to the blood exchange threshold of $50 \mu \mathrm{mol} / \mathrm{L}$ ( $3 \mathrm{mg} / \mathrm{dL}$ ), the strong phototherapy mode was adjusted to the standard phototherapy mode. While the neonate was receiving phototherapy, we applied light-shielding eye shields to cover both eyes, covered the perineum with a diaper, and exposed as much skin as possible in other areas. Strong phototherapy was first administered to neonates while preparing for blood exchange, and blood should be exchanged immediately if TSB levels did not decrease or even continued to increase, or for neonates with immune hemolysis if TSB did not decrease to 34-50 $\mu \mathrm{mol} / \mathrm{L}$ $(2-3 \mathrm{mg} / \mathrm{dL})$ after phototherapy. However, in this study, considering the principle of the $\mathrm{fMRI}$ sequence, the effect of blood exchange on functional MRI results is unknown (blood exchange may affect cerebral tissue blood flow), and we did not include neonates who received blood exchange in this study to control for population bias.

\section{Image acquisition}

Cranial MR scans of the newborn were performed within seven days of phototherapy (blue light). Specifically, MR examinations were conducted using GE Discovery MR750 3.0 T scanners (GE Healthcare, Milwaukee, WI, USA) with an eight-channel phased-array head coil with foam padding to reduce head motion. The neonate was sedated with phenobarbital $(5 \mathrm{mg} / \mathrm{kg})$ half an hour before the cranial MR examination, so that he/she could remain stationary during the examination to reduce artifacts and ensure image quality. Routine MRI included axial and sagittal spin echo T1-weighted sequences [time of repetition (TR) $400 \mathrm{~ms}$, time of echo (TE) $10 \mathrm{~ms}$ ], axial fast spin echo T2-weighted sequence (TR $3983 \mathrm{~ms}$, TE $85 \mathrm{~ms}$ ), axial T2 FLAIR sequence (TR 9,000 ms, TE $90 \mathrm{~ms}$ ), and axial diffusionweighted imaging sequence (TR 5,000 ms, TE $75 \mathrm{~ms}$, B $800 \mathrm{~s} / \mathrm{mm}^{2}$ ).

Subsequently, an echo-planar imaging sequence sensitive to $\mathrm{T} 2$ * blood oxygenation level dependent (BOLD) 
contrast material was used to acquire fMRI images with the following settings: TR 2,000 ms; TE $30 \mathrm{~ms}$; voxel size $3.1 \times 3.1 \times 3.0 \mathrm{~mm}^{3}$; flip angle $90^{\circ}$; field of view (FOV) $200 \mathrm{~mm}$; and matrix size $64 \times 64$. Each fMRI run included 160 volumes. A minimum of one run $(5 \mathrm{~min} 20 \mathrm{~s})$ was obtained for each infant. During MRI scanning, we strictly controlled the image artifacts of head movement. When the data in this sequence had image artifacts, we rescanned the sequence again until the quality of the data reached the analysis requirements.

\section{The assessment of BAEPs}

The recording of BAEPs was carried out with a Nicolet Viking IIIP machine (Nicolet Biomedical, Inc., Madison, WI, USA) at the bedside or in the neuroelectrophysiological room for neonates at the corrected age of two months. Tests were conducted by single-channel differential recording with an active electrode attached to the high forehead and a reference electrode attached to the ipsilateral mastoids. A neonatologist was responsible for BAEP testing, and BAEPs were examined with reference to previous studies that have been reported $(12,32,33)$. The results of BAEPs were interpreted by otolaryngologists and verified by pediatric neurologists. We classified the results as normal or abnormal with brainstem impairment according to the waveform data of BAEPs $(12,34)$. A test result was defined as normal when the repeatable waves $\mathrm{V}$ were detectable at less than $40 \mathrm{~dB} \mathrm{nHL}$, and latencies and interpeak latencies appeared within $2 \mathrm{SD}$ of the normal for appropriate age groups at our laboratory. Abnormal BAEPs were described as waves $\mathrm{V}$ above $40 \mathrm{~dB} \mathrm{nHL}$, and latencies of waves I, III, and $V$ exceeded 2 SD of the normal IP of I, III, and V and IPL of I-III, III-V, and I-V (32).

\section{The assessment of the neonatal long-term developmental outcomes}

We used the third version of the Bayley Scales of Infant and Toddler Development (BSID-III) to assess the developmental status of the infants at 18 months of age (18 months). The evaluation was divided into three areas: cognition, language, and body movement to systematically evaluate the development level of the subjects (35). It was confirmed that the baby had good sleep and adequate diet before the evaluation. The development evaluations of all subjects were carried out by the same evaluator, who was qualified to conduct BSID assessments. In view of the young age of the subjects, each subject was accompanied by a parent at the time of the evaluation, and the parents were advised not to give any hints to the subjects during the evaluation period. Before the evaluation, the evaluators had a simple communication with the subjects to increase their affinity, which helped to eliminate the tension of infants and give full play to the best level. If three items were passed in a row, the test was continued; otherwise, the previous starting point was selected, and the evaluation was terminated if five items failed in a row. A total of 324 test items were evaluated, including 91 items of cognition, 72 items of gross exercise, 66 items of fine exercise, 48 items of receptive communication, and 47 items of expressive communication. Among them, each item passed to get 1 point, did not pass to get 0 points, the final scores were accumulated to obtain the original score, and we calculated the composite score equivalents according to the scaled scores.

\section{Image processing and extraction of the ALFF value}

Data preprocessing was performed using SPM 8 (http://www.fil.ion.ecl.ac.uk/spm). ALFF analysis was performed using the in-house software DPARSF (Version 4.3, http:// www.restfmri.net/forum/DPARSF) $(36,37)$. The time course of each voxel was first converted to the frequency domain using a fast Fourier transformation. Then, the square root of the power spectrum was computed and averaged throughout the bandpass $(0.01-0.08 \mathrm{~Hz})$ at each voxel, that is, the ALFF. The ALFF of each voxel was divided by the global ALFF value for standardization purposes. After the color was drawn in significantly different brain areas, the standardized ALFF value reflected the degree of its raw ALFF value relative to the average ALFF value of the brain regions with the most significant difference.

\section{Statistical analysis}

Analyses were conducted using Stata version 12.0 software (Stata Corp LP, College Station, TX, USA). Differences in demographic variables were analyzed by one-way analysis of variance (ANOVA) for continuous variables and Chi-square test for categorical variables. The imaging statistical group analysis was conducted with a one-way ANOVA test to reveal the difference in the mean ALFF among the groups of three TSB concentrations. Voxel-based comparison of ALFF across the SHB, HB, and control groups was performed using one-way ANOVA in SPM8. All wholebrain voxel-wise analyses were corrected for multiple 


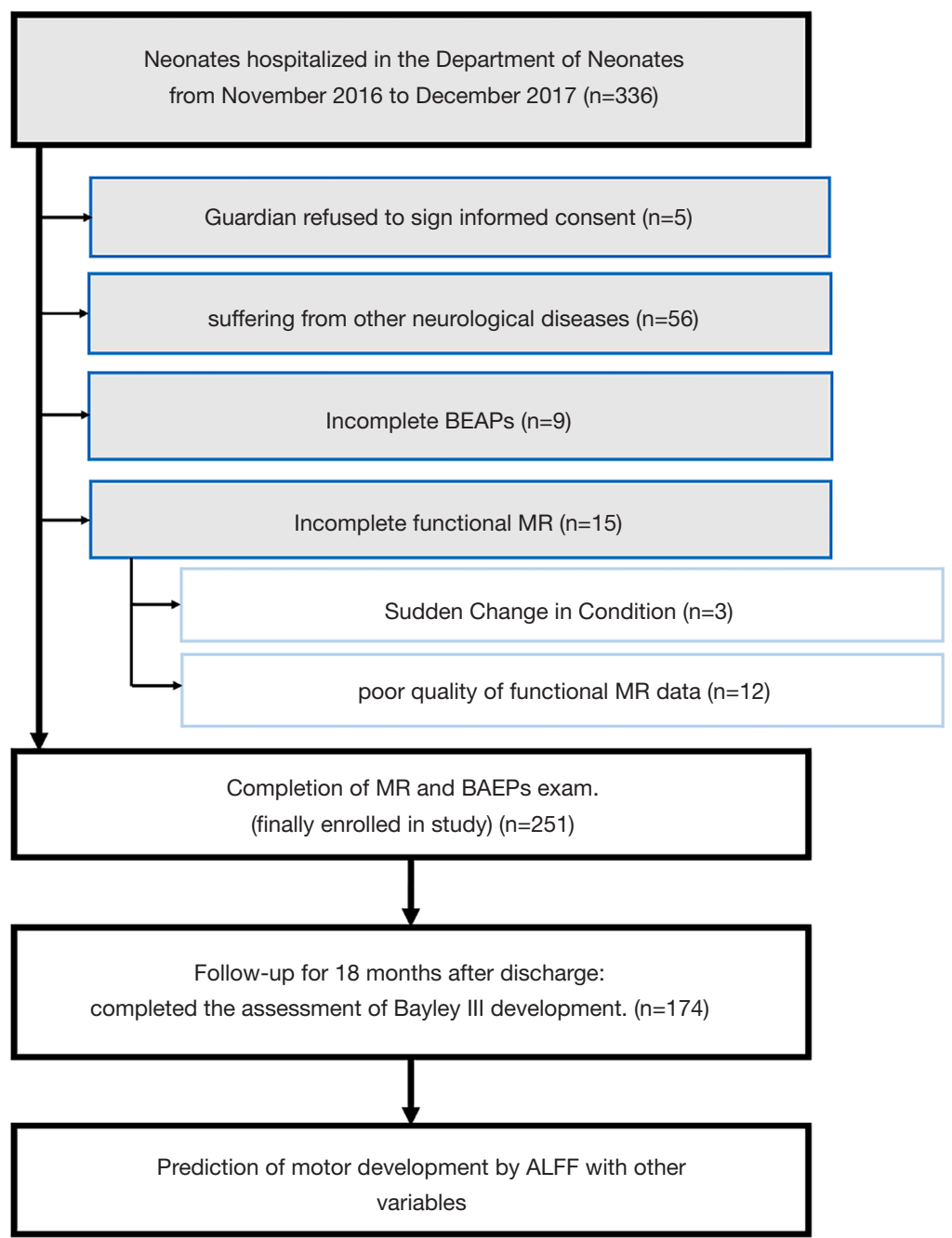

Figure 1 The flow chart of study subjects. BEAPs, brainstem auditory evoked potentials; MR, magnetic resonance; ALFF, amplitude of lowfrequency fluctuation.

comparisons to achieve a whole-brain false-positive rate of 0.05. The ALFF difference between the BSID normal group and the BSID abnormal group was compared using the independent samples $t$-test. Receiver operating characteristic (ROC) curves of TSB, ALFF, and BAEPs were plotted to conduct the area under the curve (AUC) measurements, which were used to predict the outcomes of $\operatorname{BSID}(18 \mathrm{~m})$ in neonates.

\section{Results}

\section{Participants}

Based on the inclusion and exclusion criteria, 251 newborns were enrolled in this study (Figure 1), including 45, 65, and 141 patients in the SHB, HB, and control groups, respectively. The mean gestational age was greater than 39 weeks, and the mean birth weight was greater than $3,000 \mathrm{~g}(\mathrm{P}>0.05)$. There was no difference in the sex composition of the three groups of newborns $(\mathrm{P}>0.05)$. Detailed baseline data are shown in Table 1. Of the three groups, six in the SHB group and nine in the HB group had hemolytic syndrome $(\mathrm{P}=0.001)$. All neonates with $\mathrm{HB}$ underwent a phototherapy intervention. In the SHB group, $16(35.5 \%)$ had abnormal results on conventional MRI; in the HB group, only 5 (7.7\%) had abnormal results $(\mathrm{P}<0.001)$. In the SHB group, $20(44.4 \%)$ had abnormal BAEP results; in the HB group, 6 had abnormal BAEP 
Table 1 General characteristics of all subjects

\begin{tabular}{|c|c|c|c|c|c|c|c|c|}
\hline Variable & \multicolumn{2}{|c|}{ Severe (SHB) } & \multicolumn{2}{|c|}{ Moderate (HB) } & \multicolumn{2}{|c|}{ Control } & $\mathrm{F} / \chi^{2}$ & $\mathrm{P}$ \\
\hline $\mathrm{N}$ & \multicolumn{2}{|c|}{45} & \multicolumn{2}{|c|}{65} & \multicolumn{2}{|c|}{141} & - & \\
\hline Male & \multicolumn{2}{|c|}{24} & \multicolumn{2}{|c|}{35} & \multicolumn{2}{|c|}{78} & 0.110 & 0.946 \\
\hline GA (w) & 39.3 & 1.4 & 39.7 & 1.5 & 39.6 & 1.5 & 0.671 & 0.510 \\
\hline 5 min Apgar & 8.3 & 1.1 & 8.5 & 1.1 & 8.5 & 1 & 0.351 & 0.706 \\
\hline Maternal hypertension & 7 & 17.2 & 8 & 13 & 24 & 17.6 & 0.57 & 0.752 \\
\hline Maternal diabetes & 12 & 27.6 & 14 & 22.2 & 32 & 23.1 & 0.327 & 0.849 \\
\hline Maternal thyroid dysfunction & 4 & 10.3 & 6 & 9.3 & 18 & 13.2 & 0.559 & 0.756 \\
\hline Oxygen therapy & 0 & 0 & 1 & 1.9 & 7 & 5.5 & 2.594 & 0.273 \\
\hline Hemolytic syndrome & 6 & 13.8 & 9 & 14.8 & 0 & 0 & 14.162 & 0.001 \\
\hline Elevated CRP & 4 & 10.3 & 7 & 11.1 & 20 & 14.3 & 0.476 & 0.788 \\
\hline Hepatitis syndrome & 3 & 6.9 & 3 & 5.6 & 0 & 0 & 5.766 & 0.056 \\
\hline Urinary tract infection & 7 & 17.2 & 9 & 14.8 & 23 & 16.5 & 0.104 & 0.949 \\
\hline Umbilical inflammation & 4 & 10.3 & 6 & 9.3 & 12 & 8.8 & 0.064 & 0.969 \\
\hline Phototherapy & 45 & 100 & 65 & 100 & 0 & 0 & - & - \\
\hline Abnormal routine MR results & 16 & 35.5 & 5 & 7.7 & 0 & 0 & 47.987 & $<0.001$ \\
\hline
\end{tabular}

SHB, severe hyperbilirubinemia group; HB, hyperbilirubinemia group; GA, gestational age; BW, birth weight; CRP, C reactive protein; MR, magnetic resonance; BEAPs, brainstem auditory evoked potentials.

results $(7.7 \%)(\mathrm{P}<0.001)$. A total of 10 individuals had delayed motor development: 8 in the SHB group and 2 in the HB group $(\mathrm{P}<0.001)$. The other clinical characteristics and disease composition of the three groups were balanced and comparable $(\mathrm{P}>0.05)$.

\section{ALFF of over responses to neonates with $H B$}

We compared the ALFF values of fMRI in neonates under three conditions of HB: SHB, HB, and control groups. Using one-way ANOVA analysis, we observed significant increases in ALFF in the bilateral thalamus in neonates with SHB (Figure 2A). The area at the red-yellow border is the brain region with the most significant ALFF differences between the three groups, shown in the figure (Figure 2A) as the basal ganglia region, and is involved bilaterally. In addition, the more yellow the highlighted area, the greater the ALFF difference. The most significant difference in voxel ALFF values in this region was at the location of the left thalamus. Indeed, the ALFF increased with an increase in TSB concentration under HB. A clear upward trend can be observed in the figure. Pearson correlation analysis showed that TSB was remarkably correlated with ALFF on both sides of the thalamus $\left(\mathrm{r}_{\text {thalamus }}=0.514, \mathrm{P}<0.001\right.$, Figure $2 B$ ). Among the three groups, the average value of ALFF in the SHB group was the highest, which was greater than that in the $\mathrm{HB}$ and control groups $(\mathrm{P}<0.001$, Figure 2C). 
A

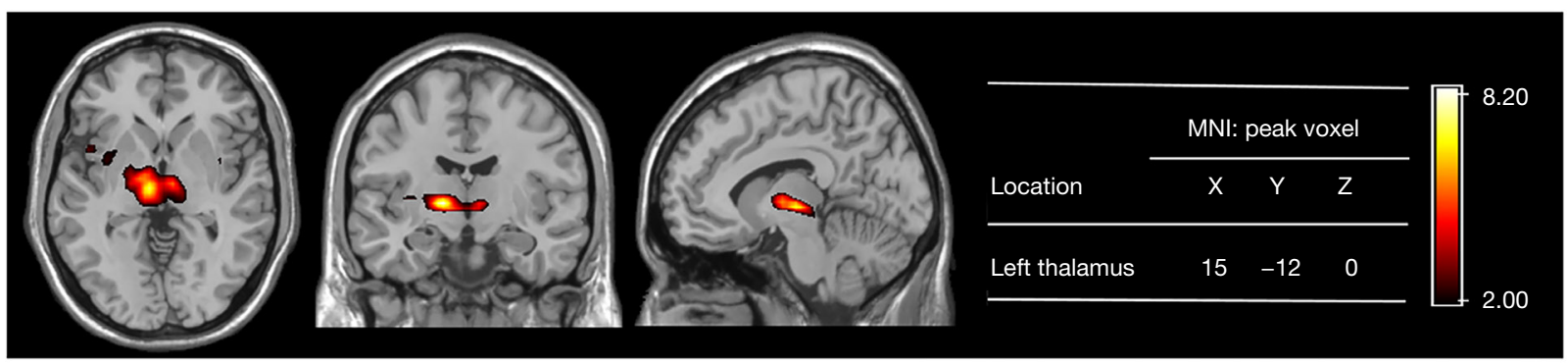

B

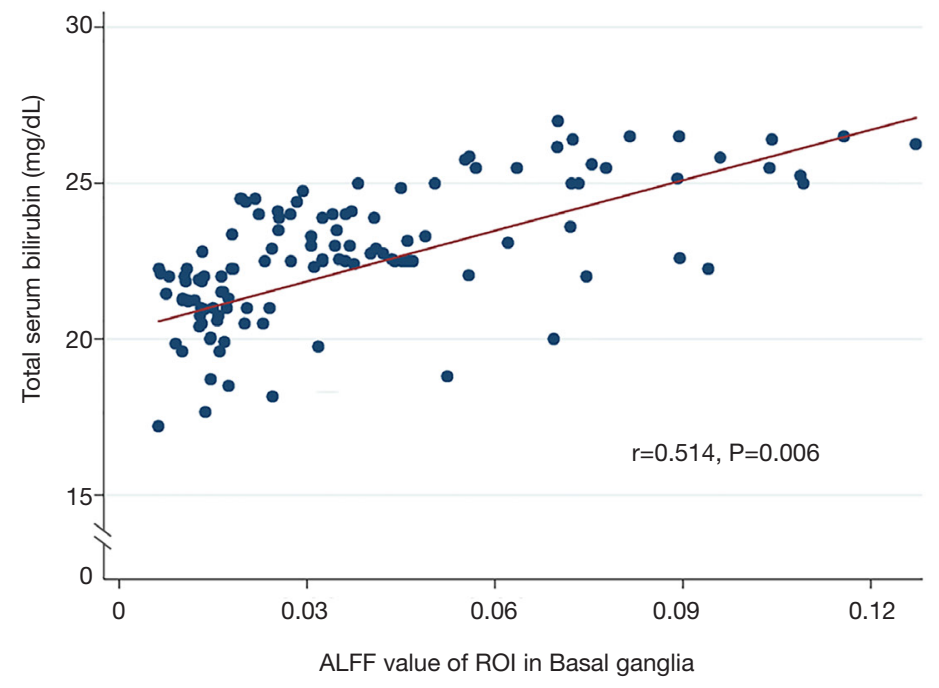

ALFF value - Linear Fitting Curve
C

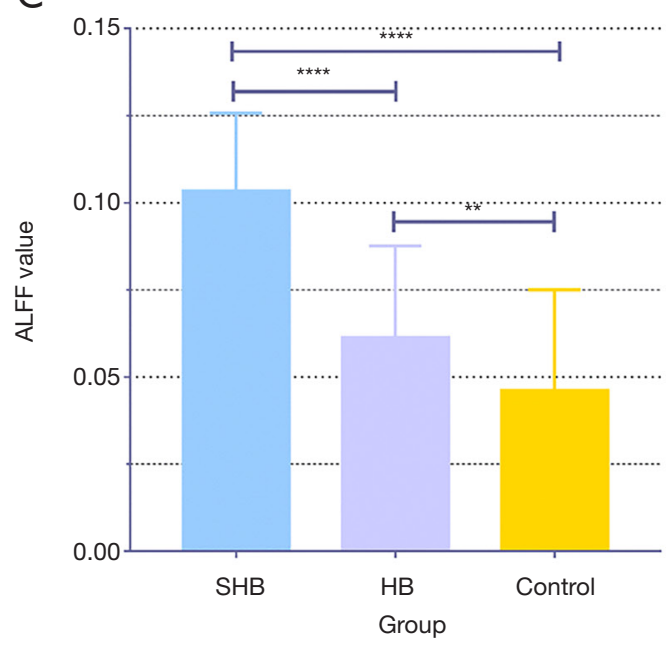

Figure 2 Comparison of ALFF values in functional magnetic resonance sequences of neonates in three groups at different serum total bilirubin levels. (A) The location of significant ALFF difference across three groups under one-way ANOVA analysis. The red and yellow highlighted area represents the area with the most significant difference in ALFF between the three groups, with the voxel with the most significant difference in ALFF located on the left thalamus. The brighter the area, the more significant the difference. (B) Correlation analysis of TSB level and ALFF value extracted from the ROI of basal ganglia. The y-axis represents the total serum bilirubin value and the $x$-axis represents the ALFF value. The red solid line represents the linear fit between ALFF and total serum bilirubin. (C) Histogram comparing ALFF values between the three groups. The blue blocks represent ALFF levels in the severe hyperbilirubin group, the purple blocks represent ALFF levels in the moderate hyperbilirubin group, and yellow shades represent ALFF levels in the control group. **, $\mathrm{P}<0.05$; ${ }^{* * * *}, \mathrm{P}<0.0001$. ALFF, amplitude of low-frequency fluctuation; TSB, total serum bilirubin; ROI, region of interesting; SHB, severe hyperbilirubinemia group; HB, hyperbilirubinemia group.

\section{Comparison of ALFF values for different findings on routine MRI}

We compared the ALFF values of the different findings on routine MRI (Table 2). In the SHB group, routine MRI showed abnormal signals in the basal ganglia or hypothalamus in 16 neonates, while there were no abnormalities in these local brain regions in the other 29 cases. In the HB group, routine MRI showed abnormal signals in the basal ganglia or hypothalamus in five neonates, while the other 60 showed no abnormalities in these local brain regions. No statistically significant differences were found when comparing the ALFF values between the two groups with normal and abnormal routine MRI findings $(\mathrm{P}>0.05)$.

\section{ALFF of over responses to neonates with low motor scores of BSID}

A total of 174 newborns completed the follow-up for BSID assessment at 18 months of age. Table 3 shows the neonates' 
Table 2 Comparison of ALFF values at different routine MR results in Neonates with hyperbilirubinemia

\begin{tabular}{|c|c|c|c|c|c|}
\hline $\begin{array}{l}\text { Hyperintensity in the basal ganglia } \\
\text { or subthalamus }\end{array}$ & $\mathrm{n}$ & \multicolumn{2}{|c|}{ ALFF value } & $t / Z$ & $P$ \\
\hline SHB MR result & & & & 1.855 & 0.070 \\
\hline Abnormal & 16 & 0.083 & 0.025 & & \\
\hline Normal & 29 & 0.065 & 0.034 & & \\
\hline Abnormal & 5 & 0.051 & 0.029 & & \\
\hline Normal & 60 & 0.046 & 0.038 & & \\
\hline
\end{tabular}

ALFF, amplitude of low-frequency fluctuation; MR, magnetic resonance; SHB, severe hyperbilirubinemia group; HB, hyperbilirubinemia group.

Table 3 Comparison of BSID scores at different TSB levels in neonates followed to 18 months of age

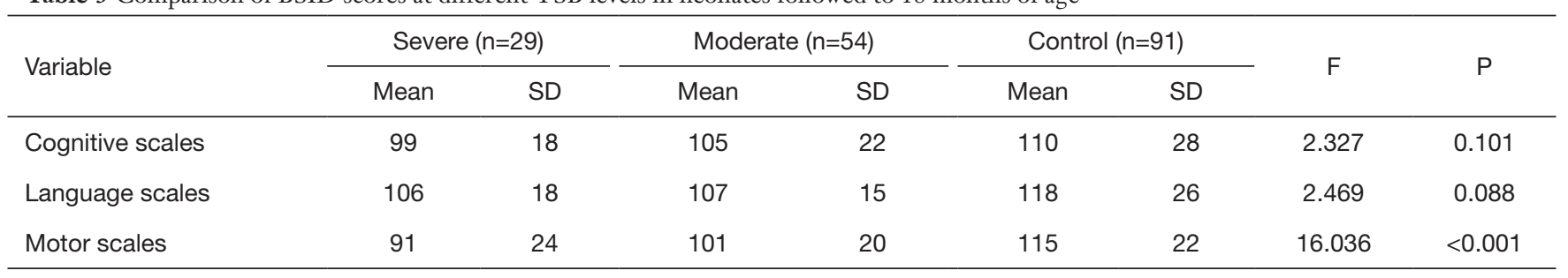

BSID, Bayley scales of infant and toddler development; TSB, total serum bilirubin.

scores on each scale after the BSID assessment. The results showed significant differences in motor development scale scores at 18 months of age among the three groups at different TSB levels $(\mathrm{P}<0.001)$. There were no statistically significant differences in cognitive and language development scale scores among the three groups $(\mathrm{P}>0.05)$.

Based on the results obtained in Table 3, we further compared the differences in ALFF values between newborns with motor development scores above 70 (including 70) and below 70, and analyzed the correlation between motor development scores and ALFF in 18-month-old neonates. By comparing the fMRI images of the two groups, we found that the most significant difference remained in the basal ganglia region (Figure 3A). The most significant areas of ALFF differences were involved in the basal ganglia region bilaterally, and the left side involved more volume than the right. In addition, the more yellow the highlighted area, the greater the ALFF difference. The most significant difference in voxel ALFF values in this region was also at the location of the left thalamus. Correlation analysis revealed a strong negative correlation between motor development scores and ALFF $(\mathrm{r}=-0.691, \mathrm{P}<0.001)$ (Figure 3B). Meanwhile, the level of ALFF was significantly lower in the normal group than in the abnormal group on the BSID assessment (Figure 3C).

\section{Prediction of the short-term motor scores in neonatal $\mathrm{HB}$}

We used four variables, including ALFF, TSB, BAEPs, and routine MRI results of the basal ganglia, to predict the incidence of motor outcomes of BSID using the ROC curve. As shown in Figure 4, when ALFF was combined with TSB, BEAPs, and routine MRI results, the area under the ROC curve was the largest (AUC $=0.85$ ). The sensitivity and specificity of the model were $67.86 \%$ and $90.77 \%$, respectively. However, when only using the ALFF model for prediction, the sensitivity can increase to $89 \%$. The specific parameters of each ROC curve are shown in Figure 4.

\section{Discussion}

Functional MRI is based on the BOLD principle $(38,39)$. Oxygen in the brain is transported to neuronal cells via hemoglobin. When neural activity increases, oxygen consumption also increases, which causes an increase in blood flow to the local area. When hemoglobin 


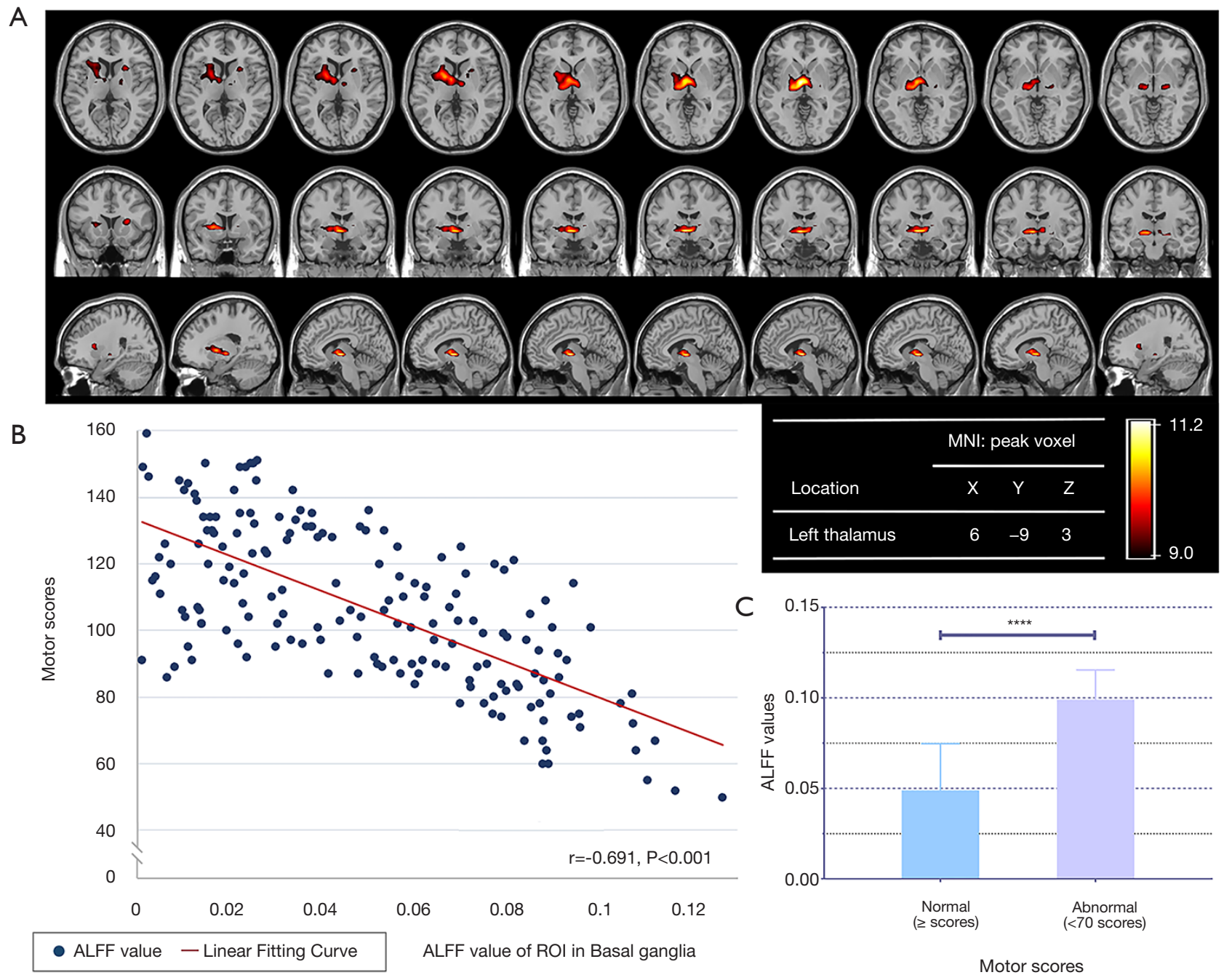

Figure 3 Comparison of ALFF values in functional magnetic resonance sequences of neonates in two groups at different BSID outcomes. (A) The location of significant ALFF difference across two groups under $t$-test analysis. The red and yellow highlighted area represents the area with the most significant difference in ALFF between the two groups, with the voxel with the most significant difference in ALFF located on the left thalamus. The brighter the area, the more significant the difference. (B) Correlation analysis of motor scores of BSID and ALFF value extracted from the ROI of basal ganglia. The $y$-axis represents the motor scores of BSID and the $\mathrm{x}$-axis represents the ALFF value. The red solid line represents the linear fit between ALFF and motor scores of BSID. (C) Histogram comparing ALFF values between the two groups. The blue blocks represent the level of ALFF in the normal group of BSID movement scale scores (greater than or equal to 70). The purple color blocks represent ALFF levels in the abnormal group (less than 70 points) of BSID Movement Scale scores. ${ }^{* * *}$, $\mathrm{P}<0.0001$. ALFF, amplitude of low-frequency fluctuation; ROI, region of interesting; BSID, Bayley scales of infant and toddler development.

binds to oxygen molecules to form oxyhemoglobin, it is antimagnetic. When oxygen molecules are free from hemoglobin, they are paramagnetic. This magnetic difference then causes a small change in the magnetic resonance signal, which is the basic principle of fMRI. In the resting state of the human body, researchers have found low-frequency electrical activity in neuronal cells even after excluding physiological noise disturbances such as respiration and heart rate (38). With the continuous development of new fMRI techniques, spontaneous neural activity in the resting state can be captured and analyzed by a number of specific methods, including ALFF, which will 

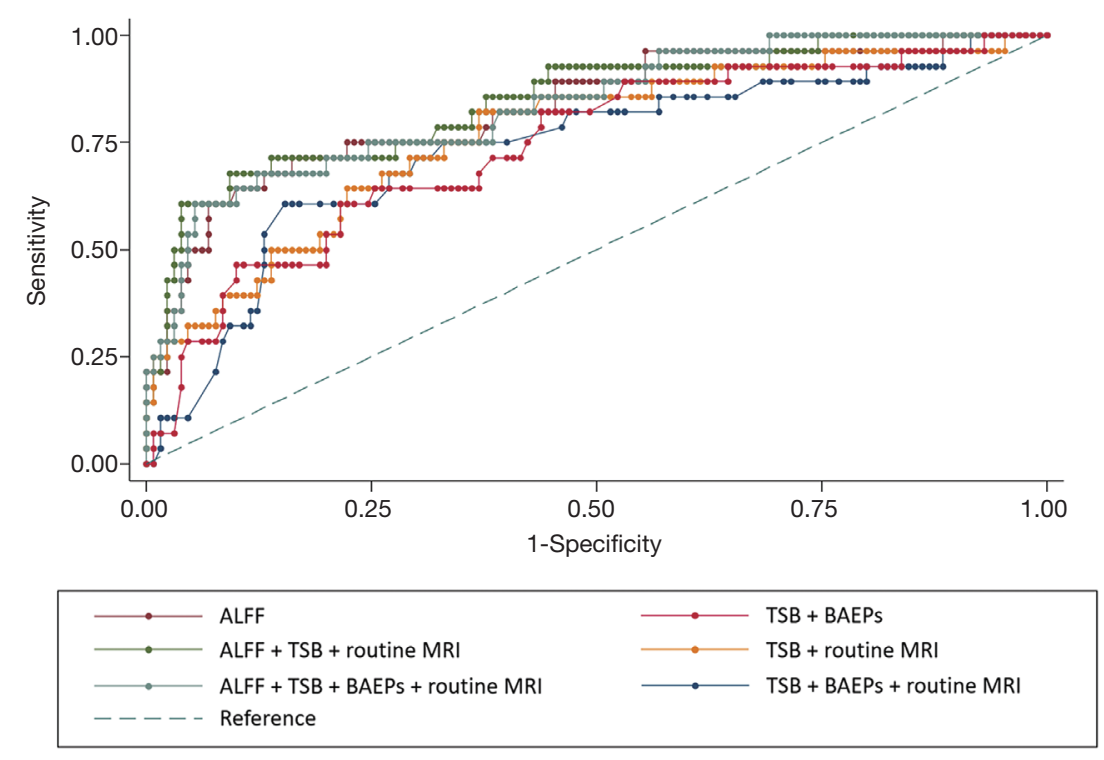

\begin{tabular}{lccccc}
\hline ROC curves & Sensitivity & Specificity & AUC & 95\% Conf. Interval \\
\hline TSB + routine MRI & $60.71 \%$ & $78.46 \%$ & 0.74 & 0.64 & 0.84 \\
TSB + BAEPs & $67.86 \%$ & $87.69 \%$ & 0.77 & 0.67 & 0.87 \\
TSB + BAEPs + routine MRI & $71.43 \%$ & $81.05 \%$ & 0.80 & 0.71 & 0.87 \\
ALFF* & $89.29 \%$ & $64.39 \%$ & 0.82 & 0.73 & 0.9 \\
ALFF + TSB + BAEPs + routine MRI & $82.14 \%$ & $63.08 \%$ & 0.84 & 0.75 & 0.92 \\
ALFF + TSB + BAEPS\# & $67.86 \%$ & $90.77 \%$ & 0.85 & 0.76 & 0.93 \\
\hline
\end{tabular}

Figure 4 Comparison of ROC curves for prediction of motor outcome by ALFF combined other covariates. In addition to ALFF values, the model incorporated three factors that previous studies have reported to be important in predicting brain damage due to hyperbilirubinemia: serum total bilirubin values, brainstem auditory evoked potential findings, and routine magnetic resonance findings (abnormal signals in the pallidum region). In total, there are five ROC curves. *, model has the highest sensitivity; *, model has the highest specificity. ALFF, amplitude of low-frequency fluctuation; TSB, total serum bilirubin; MRI, magnetic resonance imaging; BEAP, brainstem auditory evoked potential.

be discussed below.

Since Biswal et al. (38) found that spontaneous lowfrequency (nearly $0.01-0.1 \mathrm{~Hz}$ ) fluctuation (LFF) is highly synchronous among different cortices and deep gray matter, fMRI technology has been developed as a new branch of neuroimaging. ALFF (38) can be used to measure the amplitude of the spontaneous activity of local neurons. The average value of low frequency amplitude (i.e., lowfrequency signal of BOLD) during spontaneous activity is calculated to describe the spontaneous activity of neuronal cells under voxels, which reflects the level of spontaneous activity of each voxel in the resting state from the energy perspective and receives feedback from the regional activity during the resting state.

A 2013 study in FAMA Neurology reported the relationship between ALFF and cognitive outcomes in patients with severe traumatic axonal injury. The results showed that patients had greater ALFF in the frontal regions, which was correlated with cognitive performance (40). Some scholars have concluded that abnormalities in spontaneous brain activity in acute mild traumatic brain injury patients exist in the frontal lobe as well as in distributed brain regions associated with integrative, sensory, and emotional roles, and that abnormal spontaneous neuronal activity in different brain regions could be better detected by ALFF (41). Another study revealed a significant positive correlation between ALFF in the cingulate gyrus and clinical cognitive dysfunction (42) in patients with mild traumatic brain injury. In addition, longitudinal follow-up of the recovery of brain-injured patients revealed significant localized changes in ALFF compared to healthy individuals $(43,44)$. However, there is no relevant application of ALFF in the neonatal brain injury population.

Recent studies in vitro and neonatal animal models have 
found that a large number of small glial cells around the injured nerve cells are activated, or calcium deposition occurs in neurons, which may be associated with TSB induction $(8,9,45)$. The response of TSB concentration to brain injury in children is indirect, which mainly affects the nervous tissue through complex neuro-metabolic pathways. Therefore, ALFF can be used as a direct and quantitative biomarker to reflect the effect of TSB on the brain in the neonatal population. Therefore, we demonstrated that the occurrence of HB would induce an increase in ALFF in the basal ganglia, and this association would increase with the concentration of TSB. ALFF can be used to observe the effect of elevated TSB at or near the basal ganglia in neonates and quantitatively observe the changes in spontaneous neuronal activity in the gray matter nuclei around the basal ganglia.

MRI is often used clinically to observe brain injury in children with $\mathrm{HB}$ and to predict long-term prognosis (46-49). Due to the deposition of TSB, the increased signal intensity of T2-weighted imaging can be found in the basal ganglia of the affected newborn (50). The injury characteristics of near-term and full-term infants are similar, and injury to the basal ganglia area is the main target of neonatal bilirubin encephalopathy. This conclusion was also confirmed in our study. Thus, the increase in TSB may induce the signal change of basal ganglia area in neuro-images, both the neurotoxicity of unconjugated TSB in the brain and these key brain regions should be considered in the clinical diagnosis.

At present, the T1- or T2-weighted signal changes of the globus pallidus caused by the increase in TSB are the most recognized neuroimaging features in the academic community $(51,52)$. Sometimes, we can see in the routine MRI reports of newborns with $\mathrm{HB}$ that there are symmetrical abnormal signals in the pallidum and thalamic nuclei of the brain by T1- or T2-weighted imaging. In clinical practice, however, pediatricians found that neonates with T1 or T2 signal changes in the basal ganglia do not have neurodevelopmental disorders in most cases (7), and this is consistent with the results of the present study. Based on the results in Figure 2, we found that routine MRI can ignore neonates who have no obvious changes in the T1 and T2 signals. Furthermore, the routine sequence cannot quantify brain injury in the basal ganglia. Therefore, fMRI may compensate for the shortcomings of routine MRI sequences in these aspects and quantitatively demonstrate that the significantly affected brain areas were in the basal ganglia, especially the subthalamus.
Some studies have suggested that ALFF is associated with cognitive function following brain injury $(24,40,53)$. It is well known that Bayley scores can be used for the long-term assessment of neonatal neurological behavior and development. Immediately following, we analyzed the difference between the normal and abnormal groups of Bayley motor scores using the ALFF value, and found a very strong difference between these two groups. The functional connectivity of certain brain regions in the injured population is enhanced, and the ALFF values are also increased, which may be related to the compensation of nerve cells (40). In infants with low motor development scores, the changes in ALFF in the basal ganglia area of the brain are most significant. Based on ROC curves, ALFF can be a good predictor of motor development outcomes. When using ALFF combined with TSB and BAEP results to predict motor development outcomes, the AUC and specificity were the largest among all models. Therefore, when we need to synthetically predict the long-term motor development of children, we can use ALFF combined with TSB and BAEP results for better specificity. Compared with TSB or any other variables to predict motor development in neonates with $\mathrm{HB}$, the sensitivity was greatly improved by ALFF. We later found that there were patients with high ALFF who had negative MR or BAEP, as shown in Figure 2. In patients with altered ALFF, routine MR does not necessarily report positive results, and the addition of routine MR or BAEP may instead reduce the true positive rate. This also means that ALFF has a higher sensitivity compared to other examinations and can detect earlier cases reported as negative by routine MR or BAEPs, which should be the significance of ALFF.

\section{Limitations of the study}

Since the monitoring of TSB is still not dynamically available in real time, the highest bilirubin values obtained in this study may be underestimated. It is possible that some of the neonates with SHB may have been placed in the milder group. However, we still found significant differences in the ALFF values between the three groups. For the neonates to better perform the MR examination, we used a small amount of phenobarbital prior to the examination to help sedate the neonates and reduce head movement and artifacts. Although we ensured image quality, sedation may affect the baseline levels of quantitative measures such as ALFF in the fMRI sequence. However, even if it was effective, the overall trend of ALFF changes 
with the change in bilirubin will not change. We considered immature brain development in preterm infants, which may be an important confounding factor affecting ALFF levels; therefore, the study did not include the preterm population. Therefore, the results of the present study cannot be generalized to preterm infants. In addition, the scan time for routine MRI (10 $\mathrm{min})$ plus an fMRI sequence $(15 \mathrm{~min})$ takes at least $25 \mathrm{~min}$ and will be longer if head movement or artifacts are present. This may not be suitable for newborns with unstable conditions. We hope that more research will focus on this field and use a larger sample to verify our results.

\section{Conclusions}

The basal ganglia region in HB has a high ALFF value. ALFF may be an early imaging biomarker and has greater sensitivity than TSB or BAEP results in predicting longterm motor development $(18 \mathrm{~m})$ from HB. When combined with TSB and BAEP, results can be obtained with higher specificity to predict long-term motor developmental outcomes in newborns with HB. In the field of pediatrics, especially neonatology, fMRI and ALFF are still in the preliminary stage of research. There is still much research on the understanding and prognosis of bilirubin encephalopathy.

\section{Acknowledgments}

The author would like to thank all the newborns and parents who participated in this study and all the researchers who have been involved in this study for their hard work. We also thank the Audiologist Ping Lu for her guidance and help in BAEPs analysis.

Funding: This work was supported by the Leading Talents Project of Shanghai Municipal Human Resources and Social Security Bureau (Grant No. 2014-738), Program of Shanghai Subject Chief Scientist Project (Grant No. 15XD1500800) to Wenhao Zhou, the Shanghai Committee of Science and Technology, China (Grant No. 16411953400) to Guoqiang Cheng, the National Natural Science Fund of China (Grant No. 82001599) to Kai Yan, Shanghai Health and Family Planning Commission Research Project (Grant No. 20184Y0132) to Qian Qin.

\section{Footnote}

Reporting Checklist: The authors have completed the
STROBE reporting checklist. Available at http://dx.doi. org/10.21037/tp-20-447

Data Sharing Statement: Available at http://dx.doi. org/10.21037/tp-20-447

Conflicts of Interest: All authors have completed the ICMJE uniform disclosure form (available at http://dx.doi. org/10.21037/tp-20-447). The authors have no conflicts of interest to declare.

Ethical Statement: The authors are accountable for all aspects of the work in ensuring that questions related to the accuracy or integrity of any part of the work are appropriately investigated and resolved. The study protocols were registered at www.clinicaltrial.gov (NCT02544100) and were approved by the Medical Ethics Committee of Children's Hospital of Fudan University (2020-247). Written informed consent was obtained from the parents of the infant participants involved in our study. The study was conducted in accordance with the Declaration of Helsinki (as revised in 2013).

Open Access Statement: This is an Open Access article distributed in accordance with the Creative Commons Attribution-NonCommercial-NoDerivs 4.0 International License (CC BY-NC-ND 4.0), which permits the noncommercial replication and distribution of the article with the strict proviso that no changes or edits are made and the original work is properly cited (including links to both the formal publication through the relevant DOI and the license). See: https://creativecommons.org/licenses/by-nc-nd/4.0/.

\section{References}

1. Hyperbilirubinemia ASoN. Neonatal jaundice and kernicterus. Pediatrics 2001;108:763-5.

2. Dennery PA, Seidman DS, Stevenson DK. Neonatal hyperbilirubinemia. N Engl J Med 2001;344:581-90.

3. Okumura A, Kidokoro H, Shoji H, et al. Kernicterus in Preterm Infants. Pediatrics 2009;123:e1052-8.

4. Watchko JF, Claassen D. Kernicterus in premature infants: current prevalence and relationship to NICHD Phototherapy Study exchange criteria. Pediatrics 1994;93:996-9.

5. Shapiro SM, Bhutani VK, Johnson L. Hyperbilirubinemia and kernicterus. Clin Perinatol 2006;33:387-410.

6. Yan R, Han D, Ren J, et al. Diagnostic value of 
conventional MRI combined with DTI for neonatal hyperbilirubinemia. Pediatr Neonatol 2018;59:161-7.

7. Sgro M, Campbell DM, Kandasamy S, et al. Incidence of chronic bilirubin encephalopathy in Canada, 2007-2008. Pediatrics 2012;130:e886-90.

8. Yueh MF, Chen S, Nguyen N, et al. Developmental onset of bilirubin-induced neurotoxicity involves Tolllike receptor 2-dependent signaling in humanized UDP-glucuronosyltransferase1 mice. J Biol Chem 2014;289:4699-709.

9. Brites D. The Evolving Landscape of Neurotoxicity by Unconjugated Bilirubin: Role of Glial Cells and Inflammation. Front Pharmacol 2012;3:88.

10. DiPietro JA, Kivlighan KT, Costigan KA, et al. Prenatal antecedents of newborn neurological maturation. Child Dev 2010;81:115-30.

11. Streletz LJ, Graziani LJ, Branca PA, et al. Brainstem auditory evoked potentials in fullterm and preterm newborns with hyperbilirubinemia and hypoxemia. Neuropediatrics 1986;17:66-71.

12. Moreno-Aguirre AJ, Santiago-Rodriguez E, Harmony $\mathrm{T}$, et al. Analysis of auditory function using brainstem auditory evoked potentials and auditory steady state responses in infants with perinatal brain injury. Int J Audiol 2010;49:110-5.

13. Desai S, Kollros PR, Graziani LJ, et al. Sensitivity and specificity of the neonatal brain-stem auditory evoked potential for hearing and language deficits in survivors of extracorporeal membrane oxygenation. J Pediatr 1997;131:233-9.

14. Yilmaz Y, Degirmenci S, Akdas F, et al. Prognostic value of auditory brainstem response for neurologic outcome in patients with neonatal indirect hyperbilirubinemia. J Child Neurol 2001;16:772-5.

15. Fox MD. Mapping Symptoms to Brain Networks with the Human Connectome. N Engl J Med 2018;379:2237-45.

16. Thiebaut de Schotten M, Foulon C, Nachev P. Brain disconnections link structural connectivity with function and behaviour. Nat Commun 2020;11:5094.

17. Kiviniemi V, Jauhiainen J, Tervonen O, et al. Slow vasomotor fluctuation in $\mathrm{FMRI}$ of anesthetized child brain. Magn Reson Med 2000;44:373-8.

18. Seibert TM, Majid DS, Aron AR, et al. Stability of resting fMRI interregional correlations analyzed in subjectnative space: a one-year longitudinal study in healthy adults and premanifest Huntington's disease. Neuroimage 2012;59:2452-63.

19. van den Heuvel MP, Sporns O, Collin G, et al. Abnormal rich club organization and functional brain dynamics in schizophrenia. JAMA Psychiatry 2013;70:783-92.

20. Tomasi D, Volkow ND. Aging and functional brain networks. Mol Psychiatry 2012;17:471, 549-58.

21. Konova AB, Moeller SJ, Tomasi D, et al. Effects of methylphenidate on resting-state functional connectivity of the mesocorticolimbic dopamine pathways in cocaine addiction. JAMA Psychiatry 2013;70:857-68.

22. Pievani $M$, de Haan $W, W u$ T, et al. Functional network disruption in the degenerative dementias. Lancet Neurol 2011;10:829-43.

23. Chhatwal JP, Schultz AP, Johnson K, et al. Impaired default network functional connectivity in autosomal dominant Alzheimer disease. Neurology 2013;81:736-44.

24. Smyser CD, Snyder AZ, Shimony JS, et al. Effects of white matter injury on resting state fMRI measures in prematurely born infants. PLoS One 2013;8:e68098.

25. Smith SM, Beckmann CF, Andersson J, et al. Resting-state fMRI in the Human Connectome Project. Neuroimage 2013;80:144-68.

26. Neonatal jaundice and kernicterus. Pediatrics 2001;108:763-5.

27. Bhutani VK, Johnson L, Sivieri EM. Predictive ability of a predischarge hour-specific serum bilirubin for subsequent significant hyperbilirubinemia in healthy term and nearterm newborns. Pediatrics 1999;103:6-14.

28. Bhutani VK, Johnson L. A proposal to prevent severe neonatal hyperbilirubinemia and kernicterus. J Perinatol 2009;29 Suppl 1:S61-7.

29. Bhutani VK, Johnson LH, Jeffrey MM, et al. Kernicterus: epidemiological strategies for its prevention through systems-based approaches. J Perinatol 2004;24:650-62.

30. Management of hyperbilirubinemia in the newborn infant 35 or more weeks of gestation. Pediatrics 2004;114:297-316.

31. Bhutani VK. Phototherapy to prevent severe neonatal hyperbilirubinemia in the newborn infant 35 or more weeks of gestation. Pediatrics 2011;128:e1046-52.

32. Thirumala PD, Carnovale G, Habeych ME, et al. Diagnostic accuracy of brainstem auditory evoked potentials during microvascular decompression. Neurology 2014;83:1747-52.

33. Wong V, Chen WX, Wong KY. Short- and longterm outcome of severe neonatal nonhemolytic hyperbilirubinemia. J Child Neurol 2006;21:309-15.

34. Chen WX, Wong VC, Wong KY. Neurodevelopmental outcome of severe neonatal hemolytic hyperbilirubinemia. J Child Neurol 2006;21:474-9.

35. Piñon M. CHAPTER 1 - Theoretical Background and 
Structure of the Bayley Scales of Infant and Toddler Development, Third Edition. In: Weiss LG, Oakland T, Aylward GP. editors. Bayley-III Clinical Use and Interpretation. San Diego: Academic Press, 2010:1-28.

36. Zang YF, He Y, Zhu CZ, et al. Altered baseline brain activity in children with ADHD revealed by resting-state functional MRI. Brain Dev 2007;29:83.

37. Qi R, Zhang L, Wu S, et al. Altered resting-state brain activity at functional MR imaging during the progression of hepatic encephalopathy. Radiology 2012;264:187.

38. Biswal B, Yetkin FZ, Haughton VM, et al. Functional connectivity in the motor cortex of resting human brain using echo-planar MRI. Magn Reson Med 1995;34:537-41.

39. Lowe MJ, Dzemidzic M, Lurito JT, et al. Correlations in low-frequency BOLD fluctuations reflect cortico-cortical connections. Neuroimage 2000;12:582-7.

40. Palacios EM, Sala-Llonch R, Junque C, et al. Restingstate functional magnetic resonance imaging activity and connectivity and cognitive outcome in traumatic brain injury. JAMA Neurol 2013;70:845-51.

41. Zhan J, Gao L, Zhou F, et al. Amplitude of LowFrequency Fluctuations in Multiple-Frequency Bands in Acute Mild Traumatic Brain Injury. Front Hum Neurosci 2016;10:27.

42. Xiong KL, Zhang JN, Zhang YL, et al. Brain functional connectivity and cognition in mild traumatic brain injury. Neuroradiology 2016;58:733-9.

43. Sacco K, Gabbatore I, Geda E, et al. Rehabilitation of Communicative Abilities in Patients with a History of TBI: Behavioral Improvements and Cerebral Changes in Resting-State Activity. Front Behav Neurosci 2016;10:48.
44. Zou Q, Wu X, Hu J, et al. Longitudinal recovery of local neuronal activity and consciousness level in acquired brain injury. Hum Brain Mapp 2017;38:3579-91.

45. Silva SL, Vaz AR, Barateiro A, et al. Features of bilirubininduced reactive microglia: From phagocytosis to inflammation. Neurobiol Dis 2010;40:663-75.

46. Martinez-Biarge M, Diez-Sebastian J, Kapellou O, et al. Predicting motor outcome and death in term hypoxicischemic encephalopathy. Neurology 2011;76:2055-61.

47. Alderliesten T, de Vries LS, Benders MJ, et al. MR imaging and outcome of term neonates with perinatal asphyxia: value of diffusion-weighted MR imaging and $\mathrm{H}$ MR spectroscopy. Radiology 2011;261:235-42.

48. van Laerhoven $H$, de Haan TR, Offringa $M$, et al. Prognostic tests in term neonates with hypoxicischemic encephalopathy: a systematic review. Pediatrics 2013;131:88-98.

49. Massaro AN. MRI for neurodevelopmental prognostication in the high-risk term infant. Semin Perinatol 2015;39:159-67.

50. Martich-Kriss V, Kollias SS, Ball W. MR findings in kernicterus. AJNR Am J Neuroradiol 1995;16:819-21.

51. Cece H, Abuhandan M, Cakmak A, et al. Diffusionweighted imaging of patients with neonatal bilirubin encephalopathy. Jpn J Radiol 2013;31:179-85.

52. Wisnowski JL, Panigrahy A, Painter MJ, et al. Magnetic resonance imaging of bilirubin encephalopathy: current limitations and future promise. Semin Perinatol 2014;38:422-8.

53. Smyser CD, Neil JJ. Use of resting-state functional MRI to study brain development and injury in neonates. Semin Perinatol 2015;39:130-40.
Cite this article as: Yan K, Xiao F, Jiang Y, Lu C, Zhang Y, Kong Y, Zhou J, Wang J, Lin C, Yang H, Zhang D, Cheng G, Qiao Z, Wang L, Qin Q, Zhou W. Amplitude of low-frequency fluctuation may be an early predictor of delayed motor development due to neonatal hyperbilirubinemia: a fMRI study. Transl Pediatr 2021;10(5):1271-1284. doi: 10.21037/tp-20-447 